\title{
Intense high-altitude auroral electric fields - temporal and spatial characteristics
}

\author{
T. Johansson ${ }^{1}$, S. Figueiredo ${ }^{1}$, T. Karlsson ${ }^{1}$, G. Marklund $^{1}$, A. Fazakerley ${ }^{2}$, S. Buchert ${ }^{3}$, P.-A. Lindqvist ${ }^{1}$, and \\ H. Nilsson ${ }^{4}$ \\ ${ }^{1}$ Division of Plasma Physics, Alfvén Laboratory, Royal Institute of Technology, SE-100 44 Stockholm, Sweden \\ ${ }^{2}$ Mullard Space Science Laboratory, University College London, Holmbury St. Mary, Dorking, Surrey RH5 6NT, UK \\ ${ }^{3}$ Swedish Institute of Space Physics, Box 537, SE-751 21 Uppsala, Sweden \\ ${ }^{4}$ Swedish Institute of Space Physics, Box 812, SE-981 28 Kiruna, Sweden
}

Received: 30 September 2003 - Revised: 5 February 2004 - Accepted: 8 March 2004 - Published: 14 July 2004

Part of Special Issue "Spatio-temporal analysis and multipoint measurements in space"

\begin{abstract}
Cluster electric field, magnetic field, and energetic electron data are analyzed for two events of intense auroral electric field variations, both encountered in the Plasma Sheet Boundary Layer (PSBL), in the evening local time sector, and at approximately $5 R_{E}$ geocentric distance. The most intense electric fields (peaking at 450 and $1600 \mathrm{mV} / \mathrm{m}$, respectively) were found to be quasi-static, unipolar, relatively stable on the time scale of at least half a minute, and associated with moving downward FAC sheets (peaking at $\sim 10 \mu \mathrm{A} / \mathrm{m}^{2}$ ), downward Poynting flux (peaking at $\sim 35 \mathrm{~mW} / \mathrm{m}^{2}$ ), and upward electron beams with characteristic energies consistent with the perpendicular potentials (all values being mapped to $1 R_{E}$ geocentric distance). For these two events in the return current region, quasi-static electric field structures and associated FACs were found to dominate the upward acceleration of electrons, as well as the energy transport between the ionosphere and the magnetosphere, although Alfvén waves clearly also contributed to these processes.
\end{abstract}

Key words. Magnetospheric physics (electric fields; auroral phenomena; magnetosphere-ionosphere interactions)

\section{Introduction}

Intense perpendicular (to the background magnetic field) electric fields at high altitudes above the auroral region have been the subject of much interest and intense research, because they serve as an indication of electric fields parallel to the background magnetic field in the region between the $\mathrm{s} / \mathrm{c}$ and the ionosphere. One implication of upward parallel electric fields is that they will accelerate electrons down-

Correspondence to: T. Johansson

(tommy.johansson@alfvenlab.kth.se) ward, leading to auroral emissions when these particles precipitate into the upper atmosphere. The energy consumed in accelerating the auroral particles, at around $1 R_{E}$ altitude, must be available in terms of an energy flux, originating from higher altitudes. Intense perpendicular electric fields at geocentric distances of approximately $5 R_{E}$ are often found in the Plasma Sheet Boundary Layer, PSBL, which is an important source region for the energy flux powering the aurora. A fundamental question is the nature of this energy flux. Presumably, the dominant flux is an electromagnetic energy flux (Poynting flux), which can either be associated with (quasi-) static field-aligned currents (FACs) or dynamic processes, such as travelling Alfvén waves or field-line resonances (FLRs). The transport mechanism might change, so that the way by which the energy flux is carried is different at different altitudes. There is also the possibility that energy is transported as kinetic energy carried by particles, see, for example, Ostgaard et al. (2002).

Up-going (down-going) FACs are associated with negative (positive) potential structures, corresponding to converging (diverging) quasi-static electric fields. This is supported by Marklund et al. (2001), who have utilized the possibilities of the Cluster mission, consisting of four $\mathrm{s} / \mathrm{c}$, to observe the growth and decay of a diverging electric field structure accelerating electrons upward, i.e. in the return current region. (The return and primary current regions are also known as the downward and upward current regions, respectively, but the former denotation will be used here.) An increase in a positive electric field peak and the increasing energy of an upward electron beam were found to match, i.e. consistency between the parallel acceleration potential and the perpendicular potential was found, supporting the quasistatic model. However, recent studies on Polar observations (in the primary current region) have focused on the importance of Alfvén wave Poynting flux. High-altitude (above the 
Table 1. Summary of the events of 27 April and 19 May 2002. Positive (negative) $\alpha$ corresponds to anti-clockwise (clockwise) rotation of the current sheet from east-west alignment. The different $\alpha$ given for 19 May 2002 corresponds to the two different regions of spatial variations, see the text.

\begin{tabular}{|c|c|c|c|c|c|c|}
\hline Parameter/Date & \multicolumn{3}{|l|}{020519} & \multicolumn{3}{|l|}{020427} \\
\hline $\mathrm{R}\left(R_{E}\right)$ & \multicolumn{3}{|l|}{5.0} & \multicolumn{3}{|l|}{5.1} \\
\hline CGLat (degrees) & \multicolumn{3}{|l|}{-69.1} & \multicolumn{3}{|l|}{-70.5} \\
\hline MLT (h) & \multicolumn{3}{|l|}{19.7} & \multicolumn{3}{|l|}{20.0} \\
\hline$K_{p}$-index & \multicolumn{3}{|c|}{2} & \multicolumn{3}{|c|}{2} \\
\hline Substorm Phase & \multicolumn{3}{|c|}{ Peak of Expansion Phase } & \multicolumn{3}{|c|}{ Growth Phase } \\
\hline \multicolumn{7}{|l|}{$\Delta \mathrm{V}_{\perp}(\mathrm{kV})$} \\
\hline $\mathrm{s} / \mathrm{c} 1$ & \multicolumn{3}{|c|}{-0.8 and -2.2} & \multicolumn{3}{|l|}{-} \\
\hline s/c 4 & \multicolumn{3}{|c|}{-0.9 and -1.9} & \multicolumn{3}{|l|}{+2.4} \\
\hline $\mathrm{s} / \mathrm{c} 3$ & \multicolumn{3}{|c|}{-0.6 and -2.0} & \multicolumn{3}{|l|}{+2.4} \\
\hline $\mathrm{s} / \mathrm{c} 2$ & \multicolumn{3}{|c|}{-0.6 and -2.2} & \multicolumn{3}{|l|}{+2.4} \\
\hline \multicolumn{7}{|l|}{ Corresponding $\Delta \mathrm{V}_{\|}(\mathrm{kV})$} \\
\hline $\mathrm{s} / \mathrm{c} 1$ & \multicolumn{3}{|l|}{ - } & \multicolumn{3}{|l|}{$1.0-2.0$} \\
\hline $\mathrm{s} / \mathrm{c} 4$ & \multicolumn{3}{|l|}{-} & \multicolumn{3}{|l|}{$2.0-3.0$} \\
\hline $\mathrm{s} / \mathrm{c} 2$ & \multicolumn{3}{|l|}{-} & \multicolumn{3}{|l|}{-} \\
\hline $\mathrm{s} / \mathrm{c} 3$ & \multicolumn{3}{|l|}{-} & \multicolumn{3}{|l|}{$2.0-3.0$} \\
\hline Region & 1 & 2 & 3 & 1 & 2 & 3 \\
\hline $\mathrm{V}_{A}(\mathrm{~km} / \mathrm{s})$ & 2300 & 2300 & 2800 & 9000 & 5000 & 4000 \\
\hline $\mathrm{E}_{n} / \mathrm{B}_{t}(\mathrm{~km} / \mathrm{s})$ & 1800 & 3300 & 1500 & \multirow[t]{2}{*}{13000} & 18500 & 10000 \\
\hline Deviation from W-E alignment $\alpha$ (degrees) & \multicolumn{2}{|l|}{$33( \pm 5)$} & $-43( \pm 12)$ & & \multicolumn{2}{|l|}{$-30( \pm 11)$} \\
\hline
\end{tabular}

auroral acceleration region) intense electric field structures $\left(E_{\perp} \geq 100 \mathrm{mV} / \mathrm{m}\right)$ were found to be associated with large downward directed Poynting fluxes, many of which were consistent with Alfvén waves (Keiling et al., 2000, 2001, 2002, 2003; Wygant et al., 2000, 2002). The consistency of the observed structures with Alfvén waves were concluded by comparing the local Alfvén speed with the E/B-ratio of correlated electric to magnetic fields. These authors argue that the Alfvénic Poynting flux is a major contributor to the powering of the aurora, since at least one-third of the total energy required to produce the global ionospheric auroral luminosity can be accounted for by Alfvén waves (Keiling et al., 2003). Also, a relation between the large Alfvénic Poynting fluxes and the expansion phase of both strong and weak substorms was found by Keiling et al. (2000, 2001). However, the observations reported by Keiling et al. (2001) are consistent with both the quasi-static model and dynamic Alfvén wave processes. In a FAST/Polar conjunction study, Schriver et al. (2003) have found intense electric fields associated with Alfvén waves in the primary current region and shown in several events that both FAC and Alfvén waves transport energy into the auroral region, with the presence of the Alfvén waves depending on the geomagnetic activity (no Alfvén waves during periods of low activity). The above referred to Polar studies (primary current region), this study, as well as Marklund et al. (2001) (return current region), are studies, on parts of the same current system, connected via perpendicular currents in the ionosphere and driven by a generator, presumably in the magnetosphere.
A special case of Alfvén wave phenomena is FLRs. These have electric and magnetic field topologies similar to those of discrete auroral arcs, hence FLRs have been proposed as a producer of aurora. There is observational support for this proposition, see, from example, Samson et al. (1996, 2003). Further, Lotko et al. (1998) found that their FLR model can reproduce the large-scale field structure of an auroral acceleration region.

Two events of intense electric fields observed in the auroral return current region in the evening sector, from April and May 2002, have been studied using Cluster data. They are connected to the PSBL, as deduced from PEACE (Plasma Electron And Current Experiment) electron and CIS (Cluster Ion Spectrometry) ion data. For descriptions of the Cluster instruments, see Escoubet et al. (1997). The s/c are for both events located at a geocentric distance of approximately $5 R_{E}$. The geomagnetic activity is low or moderate, inferred from the $K_{p}$-index, and both events are in the Southern Hemisphere.

The intent of this paper is to describe the two events and to present a view of the stability or variations of some relevant parameters, such as electric and magnetic fields, Poynting fluxes, FACs, the potentials along the spacecraft trajectory, and electron and ion distributions. The capability of simultaneous measurements provided by the four closely spaced Cluster s/c allows us to reveal whether variations are temporal or (and) spatial. The method used for separating spatial and temporal variations in the electric and magnetic fields is described by Karlsson et al. (2004) in this issue 
(hereafter called the "companion paper"). The variations of some parameters and the stability of others can, together with a knowledge of whether the variations are spatial or temporal, improve the understanding of the different means of energy transport between the magnetosphere and the ionosphere. More specifically, the relative roles of the FACs and the Alfvén waves in this process are investigated.

\section{Observations of intense electric fields}

The criterion used when selecting the events was the presence of intense electric fields mapping to at least $100 \mathrm{mV} / \mathrm{m}$ at ionospheric altitude. Further, a similar electric field pattern should be recognized by at least two s/c. Twenty-four events of intense electric fields that fulfilled the selection criteria were found in the period January to June 2002. Two of these were chosen to be studied in more detail. The selection was based on the characteristics of the events and on legible data. In this section, data for the two events are presented and discussed. The results are summarized in Table 1.

\subsection{May 2002}

On 19 May 2002, 05:26-05:36 UT, the Cluster s/c were at a geocentric distance of $5.0 R_{E}$, around -70 CGLat and close to $20 \mathrm{MLT}$, i.e. in the evening local time sector and in the Southern Hemisphere. This event took place at the peak of a substorm expansion phase (the auroral oval at its maximum expanded state, revealed by a maximal peak in the AL index, after which a decrease begins, corresponding to the recovery phase), with moderate geomagnetic activity (a $K_{p}$-index of $2)$. Figure 1 gives the configuration of the four $\mathrm{s} / \mathrm{c}$, the direction of motion of the $\mathrm{s} / \mathrm{c}$ and the orientation of the current sheets (see below) in a plane perpendicular to the background magnetic field.

Using the method described in the companion paper to solve the temporal and spatial ambiguity problem, three regions of different electric and magnetic characteristics could be identified; 200-300s, 300-380s and 380-440s after 05:26 UT. (See Figs. 2-5, described below.) In regions I and III, similar features in the electric and magnetic field data where observed by the consecutive s/c with a time lag of approximately $10 \mathrm{~s}(1-15 \mathrm{~s})$. No clear correlations between the electric and magnetic fields are observed when looking at the data. ("Correlation", used here and later, does not refer to a calculated correlation but to a clear similarity between a parameter observed by consecutive s/c or between different parameters observed by the same s/c.) The Alfvén velocities (calculated assuming, for simplicity, equal amounts of hydrogen and oxygen ions and the plasma density estimated from the s/c potential) are 2300 and $2800 \mathrm{~km} / \mathrm{s}$, respectively, and the $E_{n} / B_{t}$-ratios (see discussion of normal and tangential direction below) are 1800 and $1500 \mathrm{~km} / \mathrm{s}$, respectively. Note that there are relatively large uncertainties in these estimates such that the Alfvén velocity and the $E_{n} / B_{t}$-ratio may differ by as much as a factor of two, even for the case of

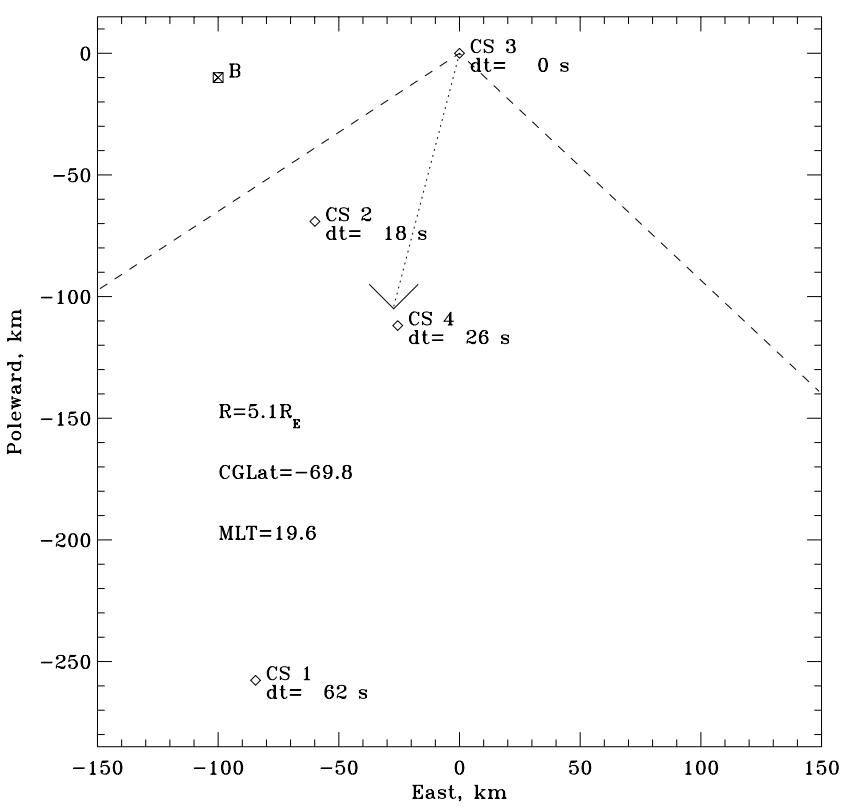

Fig. 1. 19 May 2002. The direction of motion (dotted line) and configuration of the $\mathrm{s} / \mathrm{c}$ in a plane perpendicular to the background magnetic field. $\mathrm{dt}$ is the time separation of the satellites passing through this plane, with respect to s/c 3 . The orientations of the current sheets (see the text) are shown as dashed lines.

clear Alfvén waves. In regions I and III, the variations are concluded to be predominantly spatial and corresponding to moving field-aligned current sheets having peaks coinciding with the electric field peaks. From the separations between the $\mathrm{s} / \mathrm{c}$ and the observations of the current sheets, it is concluded that the current sheets are moving with a fairly constant angle of deviation from east-west alignment within each of the two regions. The current sheet orientations obtained are not the same in regions I and III. The deviations from east-west alignment, $\alpha$, were approximately 34 and $-43^{\circ}$, respectively (determined by a minimum variance analysis on the residual magnetic field), and the current sheet velocity perpendicular to this orientation was 8 and $14 \mathrm{~km} / \mathrm{s}$, respectively. Positive (negative) $\alpha$ corresponds to an anti-clockwise (clockwise) rotated current sheet with respect to the eastwest orientation. The order in which the s/c encountered the current/electric field structures was 1, 4, 2 and 3 and the satellite data are presented in this order. The time difference between the structure crossings in region I by s/c 1 and by s/c 4, 2 and 3 were approximately 1, 7 and $12 \mathrm{~s}$. In region III, the time differences between the crossings of the spatial structures were 6,9 and $13 \mathrm{~s}$, respectively. There is a large-scale upward current of Region 1 type between 100 and $200 \mathrm{~s}$, and a large-scale downward current of Region 2 type between 200 and $290 \mathrm{~s}$ which coincides with region I of this event. After $300 \mathrm{~s}$, for regions II and III of this event, the large-scale current consists of both upward and downward currents. In region II, the electric and magnetic field data are closely correlated with almost no time lag, indicating that temporal variations are dominating there. The calculated 

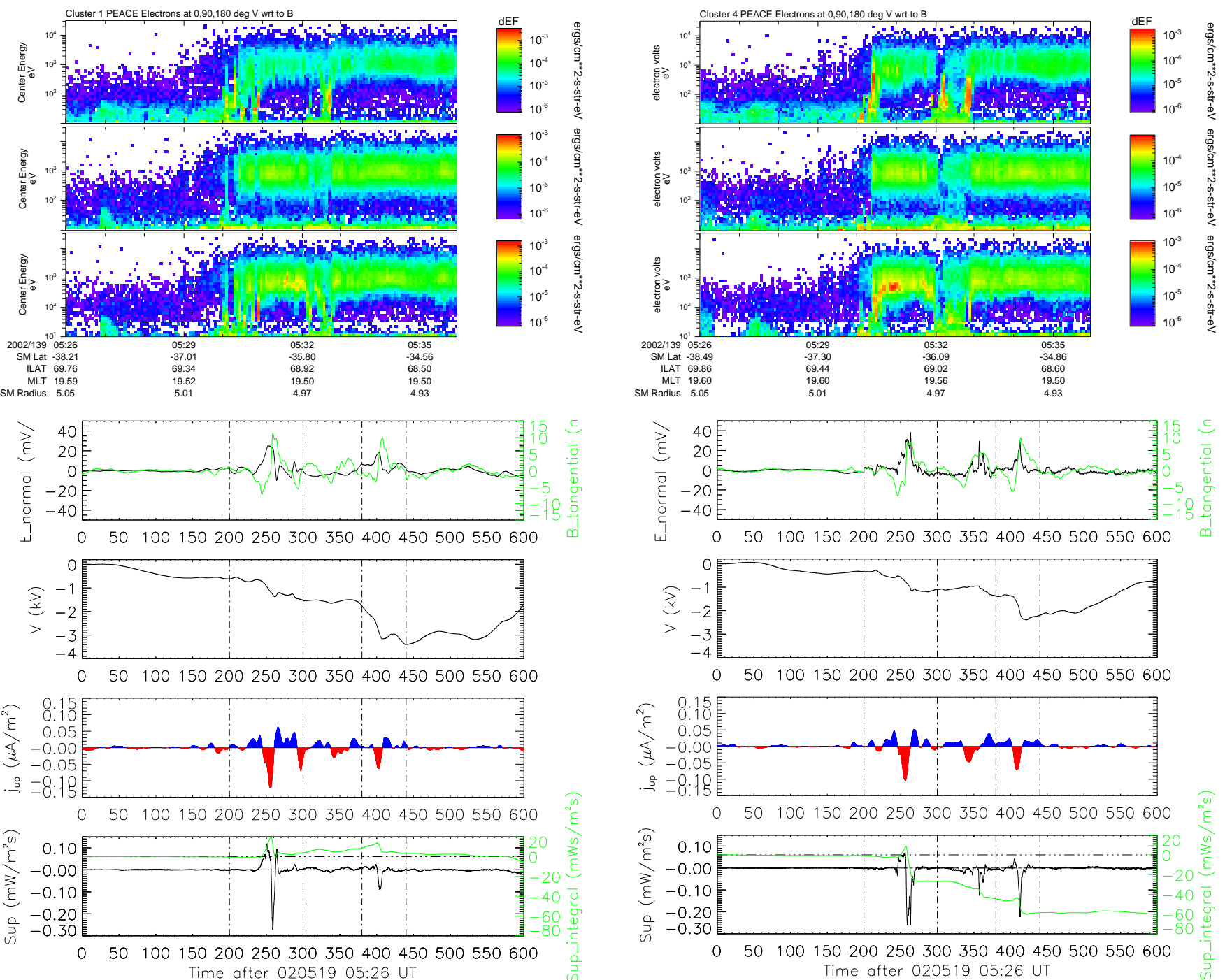

Fig. 2. 19 May 2002, s/c 1. Electron energy-time spectrograms at pitch angles 0,90 and $180^{\circ}$ are shown in the first three panels. This event is on the Southern Hemisphere, hence the first panel shows electrons going upward from Earth and the downward electrons are seen in the third spectrogram panel. The following panel shows the component of the electric field normal to the current sheet (black line) and the tangential component of the magnetic field (green line). Only these two components are shown, since they are the ones where the dominating variations are seen. The next panel displays the potential along the spacecraft trajectory. A negative (positive) value in the second to last panel corresponds to downward (upward) FACs and is shown with red (blue) color. Poynting flux (black line) and the integrated Poynting flux (green line) are plotted in the bottom panel. The vertical dash-dot lines delimit the regions of dominating spatial and temporal variations (see the text). All panels are local value plots, with the ionospheric mapping factors being 11.2 for E and 125 for FAC and S.

local Alfvén velocity, $2300 \mathrm{~km} / \mathrm{s}$, agrees roughly with the local $E_{n} / B_{t}$-ratio of $3300 \mathrm{~km} / \mathrm{s}$, considering the uncertainties in the calculations caused by the rough estimate of the ion composition. The frequencies of these Alfvén waves are in
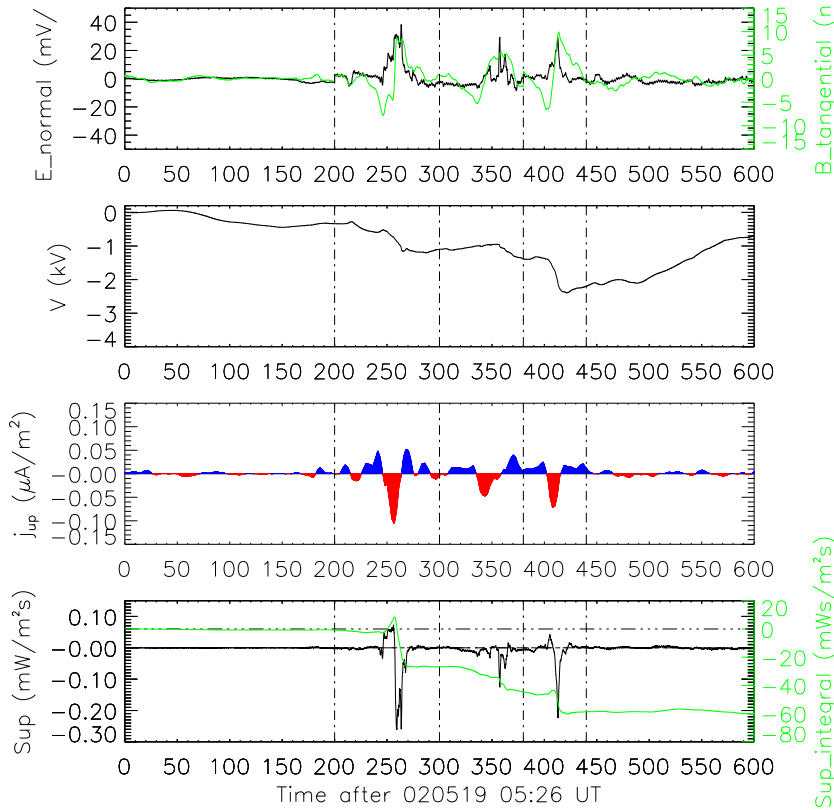

Fig. 3. 19 May 2002, s/c 4. The same panels as in Fig. 2.

the range $25-50 \mathrm{mHz}$, somewhere in between those of FLRs and those associated with the Alfvénic resonator. A closer investigation of the variations can be found in the companion paper.

S/c 1: At the top of Fig. 2, electron data from the PEACE instrument are shown in the first three panels. The electron data are in the form of energy-time spectrograms at pitch angles of 0,90 and $180^{\circ}$, up-going electrons are seen in the first panel and downward flowing electrons in the third panel. Note the different scales for up-going and down-going electron fluxes. The electric and magnetic fields were projected into a local coordinate system determined by the direction of minimum variance in the residual magnetic field. If the encountered variations are predominantly spatial, as concluded to be the case in regions I and III, the direction of minimum variance in the residual magnetic field is tangential to the current sheet. Panel 4 shows the electric field component normal to (what in regions I and III can be interpreted as) the local current sheet orientation (black line) and the magnetic 

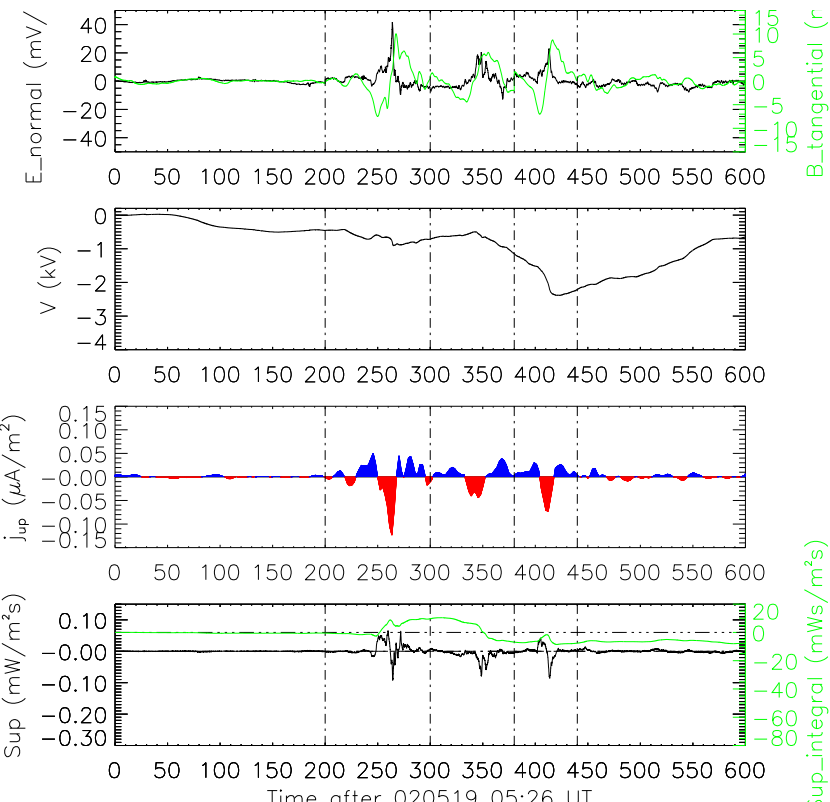

Fig. 4. 19 May 2002, s/c 2. The same panels as in Fig. 2 but without PEACE electron data.

field component tangential to the local current sheet orientation (green line). The electric field was measured by the EFW (Electric Field and Wave) instrument and the magnetic field by the FGM (FluxGate Magnetometer) instrument. Due to a probe failure, only spin resolution electric field data are available from $\mathrm{s} / \mathrm{c} 1$. From the measured magnetic field data, a running-window average (1-min wide and steps of $10 \mathrm{~s}$ ) was subtracted to remove the background field. The potential along the spacecraft trajectory was obtained by integrating the electric field, and is presented in panel 5. Panel 6 shows the FAC distribution estimated from the residual magnetic field (assuming spatial magnetic variations). The calculated FAC is unreliable in regions where the variations were found to be predominantly temporal due to Alfvén waves, such as in region II. A negative (positive) value corresponds to downward (upward) FACs and is shown with red (blue) color. From the electric field and the residual magnetic field data, the Poynting flux along the background magnetic field, $S_{\|}$, was calculated and is displayed in the bottom panel (black line), together with the time integrated Poynting flux (green line). The data presented are all local values and in order to refer to the ionospheric level, the mapping factors are given in the text below Fig. 2 .

Two peaks in the electric field data, one in each of the two regions dominated by spatial variations, are seen at 260 and $400 \mathrm{~s}$. Their magnitudes are, if mapped to the ionosphere, 280 and $200 \mathrm{mV} / \mathrm{m}$, respectively. Two downward FAC peaks and two downward Poynting flux peaks are well correlated with the electric field peaks. At $260 \mathrm{~s}$, the magnitude of the mapped downward Poynting flux peak is $33 \mathrm{~mW} / \mathrm{m}^{2}$ and the magnitude of the mapped downward FAC (this estimate depends on the filtering of the magnetic field and should be
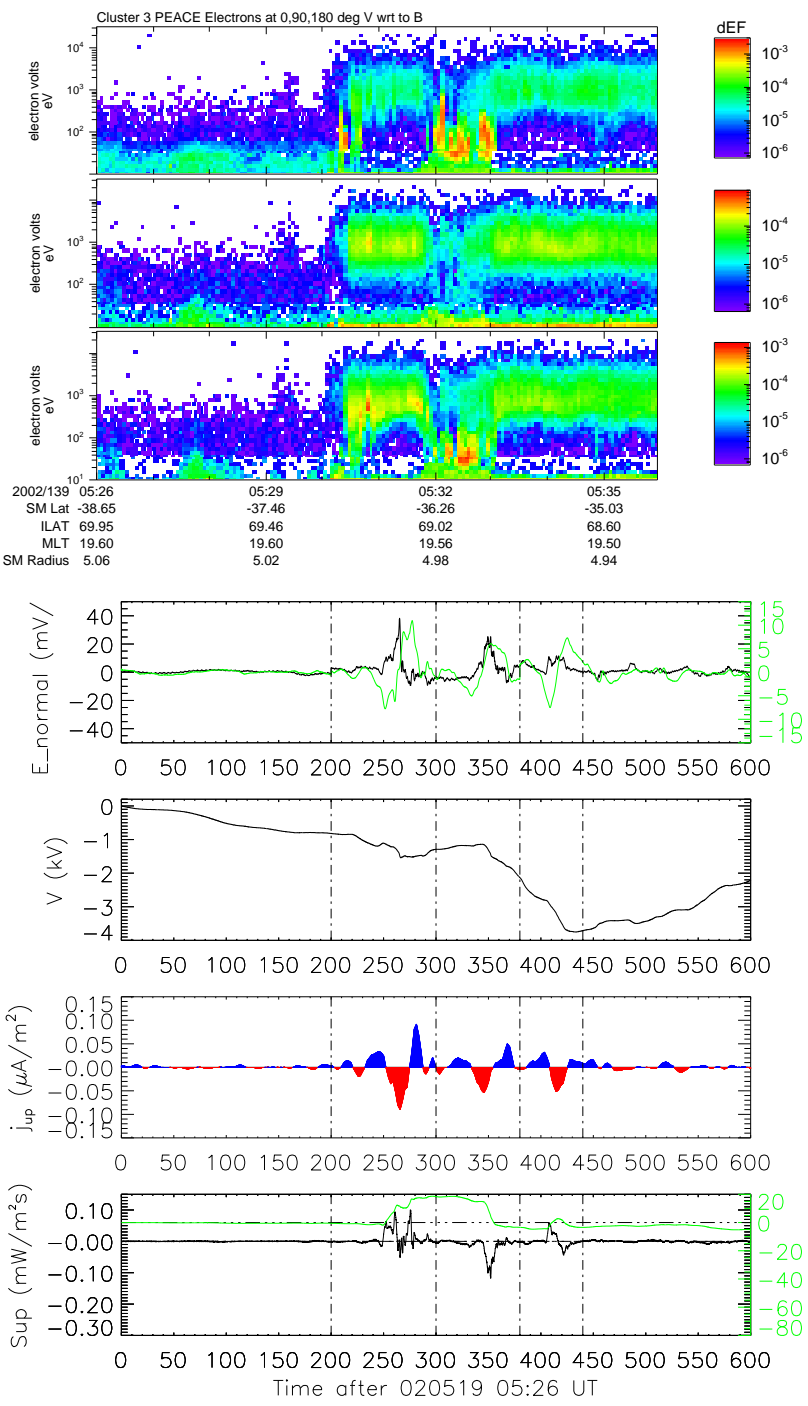

Fig. 5. 19 May 2002, s/c 3. The same panels as in Fig. 2.

considered with care; this study is mostly concerned with the variations between consecutive s/c) is $15 \mu \mathrm{A} / \mathrm{m}^{2}$. The Poynting flux is downward dominated but the integrated Poynting flux is close to zero. The remaining parameter, the perpendicular potential, is seen to display a large-scale negative valley structure. There are also superposed small-scale valley shapes between 400 and $470 \mathrm{~s}$, the total minimum being $-3.4 \mathrm{kV}$. By comparison with CIS data (not shown here) it was found that the small-scale negative potentials agreed roughly with the characteristic energy of the up-going ions. From inspection of the PEACE electron data (a sharp increase in the electron flux, supported by CIS data showing also an ion flux increase) for this and the other s/c passages, it is concluded that the $\mathrm{s} / \mathrm{c}$ are traversing the PSBL.

S/c 4: Three distinct more or less unipolar electric field structures, one in each region, are seen in the data from the passage by s/c 4 (Fig. 3), with the most intense peak at $250 \mathrm{~s}$ mapping to $420 \mathrm{mV} / \mathrm{m}$ at ionospheric altitude. The peak in the middle region was not present in the electric field data 


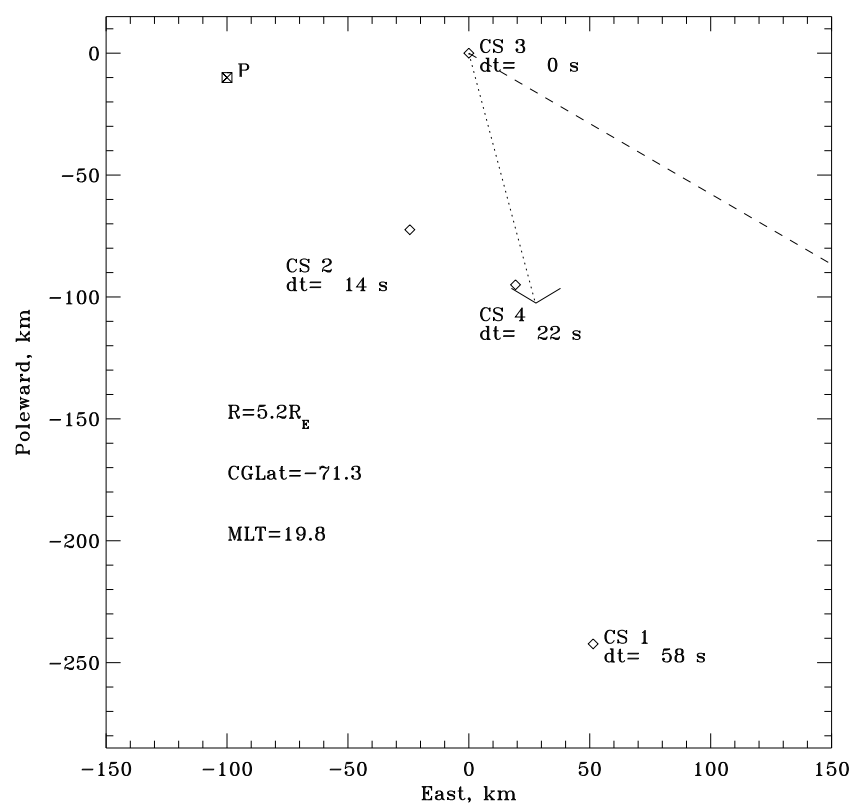

Fig. 6. 27 April, 2002. The direction of motion (dotted line) and configuration of the $\mathrm{s} / \mathrm{c}$ in a plane perpendicular to the background magnetic field. $d t$ is the time separation of the satellites passing through this plane, with respect to s/c 3 . The orientation of the current sheet (see the text) is shown as a dashed line.

from the passage by s/c 1 . Three distinct regions of intense up-going electrons with characteristic energies of $2-3 \mathrm{keV}$, not seen in the data observed by s/c 1, are seen well separated, with the first and the last of these being well correlated with the downward FAC peaks at 260 and $410 \mathrm{~s}$ (the FAC pattern is fairly similar to that observed by s/c 1 ), and also with the intense electric field peaks and the downward Poynting flux peaks. The Poynting flux is in this passage more downward dominated, due to more downward contributions from the two peaks at 260 and $410 \mathrm{~s}$. The magnitude of the later peak has increased to $25 \mathrm{~mW} / \mathrm{m}^{2}$, all compared to the observations by $\mathrm{s} / \mathrm{c} 1$. In the region dominated by temporal variations, a downward Poynting flux peak is observed in contrast to what was observed in the passage by s/c 1 . Finally, the depth of the large-scale potential valley in the later part of the interval has decreased to $-2.4 \mathrm{kV}$. Note that the negative valley in the perpendicular potential pattern between 250 and $350 \mathrm{~s}$ coincides well with the inverted V-structure in the precipitating electrons, implying that these electrons will be subject to further acceleration by the order of $1 \mathrm{kV}$ before they reach the auroral ionosphere.

S/c 2: The large-scale, electric field pattern, with the peak at $260 \mathrm{~s}$ (mapping to $450 \mathrm{mV} / \mathrm{m}$ ), and the FAC pattern are both roughly the same as for the passage by $\mathrm{s} / \mathrm{c}$ 4 (Fig. 4). The Poynting flux has decreased significantly (the main downward peak has decreased from $33 \mathrm{~mW} / \mathrm{m}^{2}$ to $12 \mathrm{~mW} / \mathrm{m}^{2}$ ) and the integrated Poynting flux is small but negative. However, the correlation between the intense electric field peaks, the downward FAC peaks and the downward Poynting flux peaks, at approximately 260 and 400 s, per-
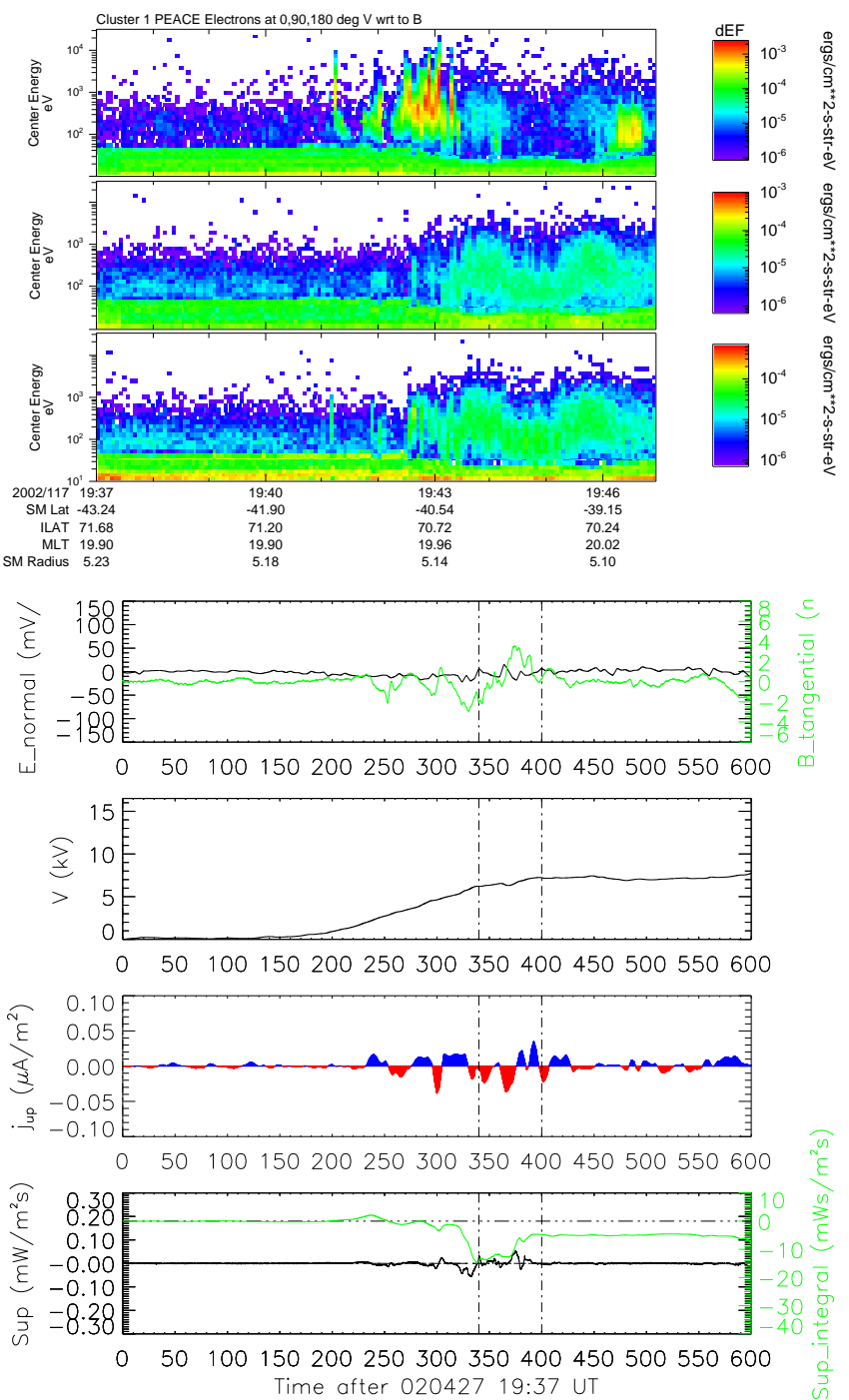

Fig. 7. 27 April 2002, s/c 1. The same panels as in Fig. 2. The vertical dash-dot lines delimit the region of dominating spatial variations (see the text).

sists. No significant variations are observed in the perpendicular potential. Neither PEACE nor CIS data were available from $\mathrm{s} / \mathrm{c} 2$.

S/c 3: The electric and magnetic field patterns are very similar to those of the previous $\mathrm{s} / \mathrm{c}$ crossings, although the third electric field peak at $410 \mathrm{~s}$ in region III has decreased in magnitude (Fig. 5). Thus, the FAC pattern is also fairly unchanged but the downward peak around 260 is now seen to be somewhat broader. The enhancements in the up-going electron flux (more intense than for s/c 4), with characteristic energies of 2-3 keV, are consistent with the downward FAC peaks and well correlated with the electric field peaks in regions I and III.

In region II, the downward peak of the Poynting flux dominated by temporal variations is seen to have increased to $12 \mathrm{~mW} / \mathrm{m}^{2}$, locally, while the Poynting flux in regions I and III is similar to the corresponding Poynting flux in the passage by s/c 2 but not to the fluxes observed by s/c 1 and 4 . 


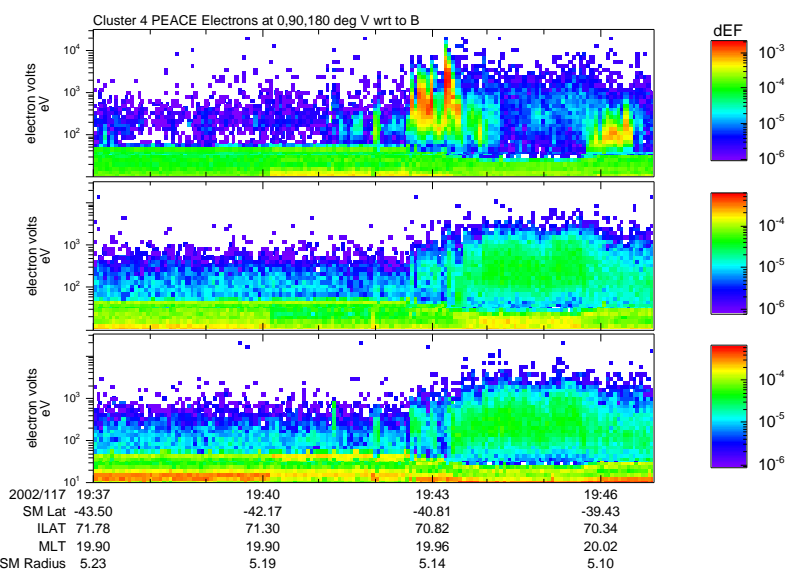

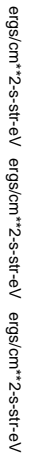
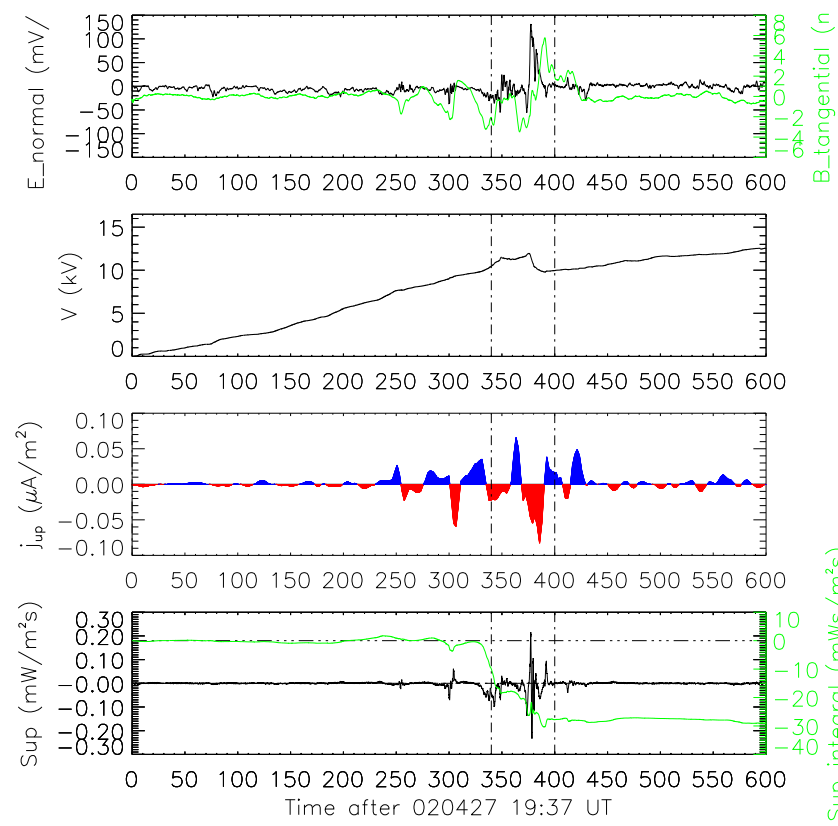

Fig. 8. 27 April 2002, s/c 4. The same panels as in Fig. 2.

It can also be seen that the potential profile differs from the two previous crossings in that the depth of the large-scale negative perpendicular potential structure has increased from $-2.4 \mathrm{kV}$ to $-3.7 \mathrm{kV}$ and is resembling the profile observed by s/c 1 .

\section{$2.2 \quad 27$ April 2002}

For the event of 27 April 2002 (19:37-19:47) the Cluster $\mathrm{s} / \mathrm{c}$ were at a geocentric distance of $5.1 R_{E}$, close to $20 \mathrm{MLT}$ and around -71 CGLat, in the Southern Hemisphere (Fig. 6). The $K_{p}$-index for this event was 2, indicating moderate geomagnetic activity, and from the AL-index it is concluded that the measurements were taken during the growth phase of a substorm.

Based on the method described in the companion paper, applied to this event, and on the characteristics of the electric and magnetic field data, and the electron flux data (see Figs. 7-10), it is found that the region of predominantly spatial variations encompasses the entire upward electron beam
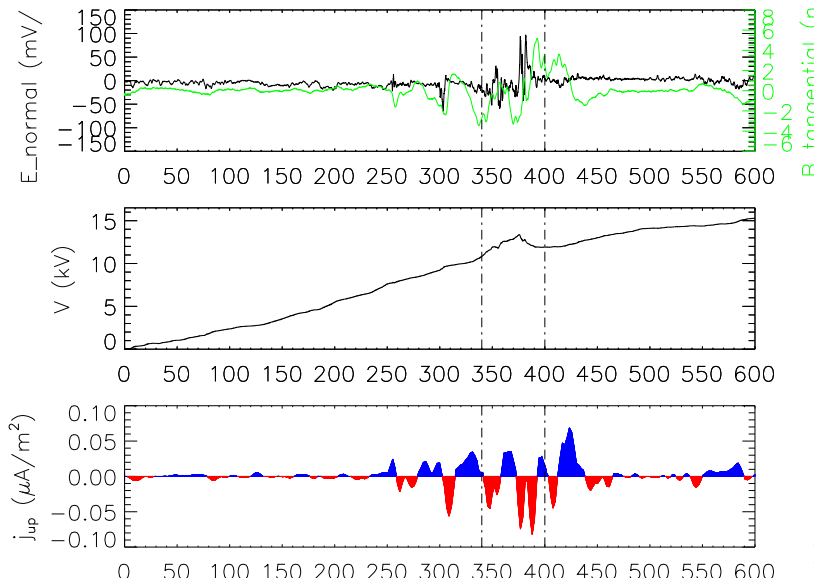

o 50100150200250300350400450500550600

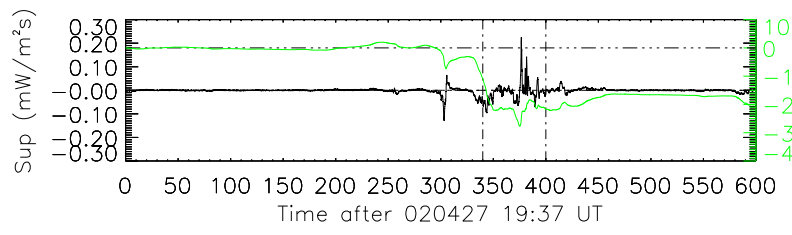

Fig. 9. 27 April 2002, s/c 2. The same panels as in Fig. 2 but without PEACE electron data.

structure, i.e. between 340 and $400 \mathrm{~s}$ after 19:37 UT. Inspection of the various data affirms that spatial variations are dominating in this whole region, although the strongest support to the interpretation that the variations are of a quasistatic nature is obtained for the later half of this interval. The deviation from east-west alignment, $\alpha$, was $-30^{\circ}$, and the structure had a velocity perpendicular to this orientation of $3.7 \mathrm{~km} / \mathrm{s}$. The order by which the s/c encountered the current/electric field structure was 1, 4, 2 and 3 and the time differences between the crossings of the structure were approximately 14,16 and $23 \mathrm{~s}$, respectively. A large-scale downward current of Region 2 type between 300 and 400 s coincides with the downward current in this event. Starting approximately $100 \mathrm{~s}$ before this region, an upward current of Region 1 type is seen.

S/c 1: The electric field is small over the whole interval (Fig. 7) and so are the magnitudes of the predominantly downward Poynting flux. The FAC pattern is characterized by multiple and weak up- and down-going currents. A region of downward FAC between 340 and $380 \mathrm{~s}$ is correlated with enhanced upward electron flux, with characteristic energies of about $1 \mathrm{keV}$. Overlapping this region, the perpendicular potential increases during the first $400 \mathrm{~s}$ after 19:37 UT, and then remains constant at $7.5 \mathrm{kV}$. From inspection of the PEACE electron data for all four passages, it was concluded that the $\mathrm{s} / \mathrm{c}$ were in the PSBL.

S/c 4: At the time of the crossing by s/c 4 (Fig. 8) the electric field has intensified, the magnitude of the main peak at $380 \mathrm{~s}$ is now $1500 \mathrm{mV} / \mathrm{m}$, mapped to the ionosphere. The strongest variations in both the electric and magnetic fields are found to be correlated with intensifications in the upward electron flux, downward FAC and intense Poynting flux and are identified to be clearly of a quasi-static nature. The 

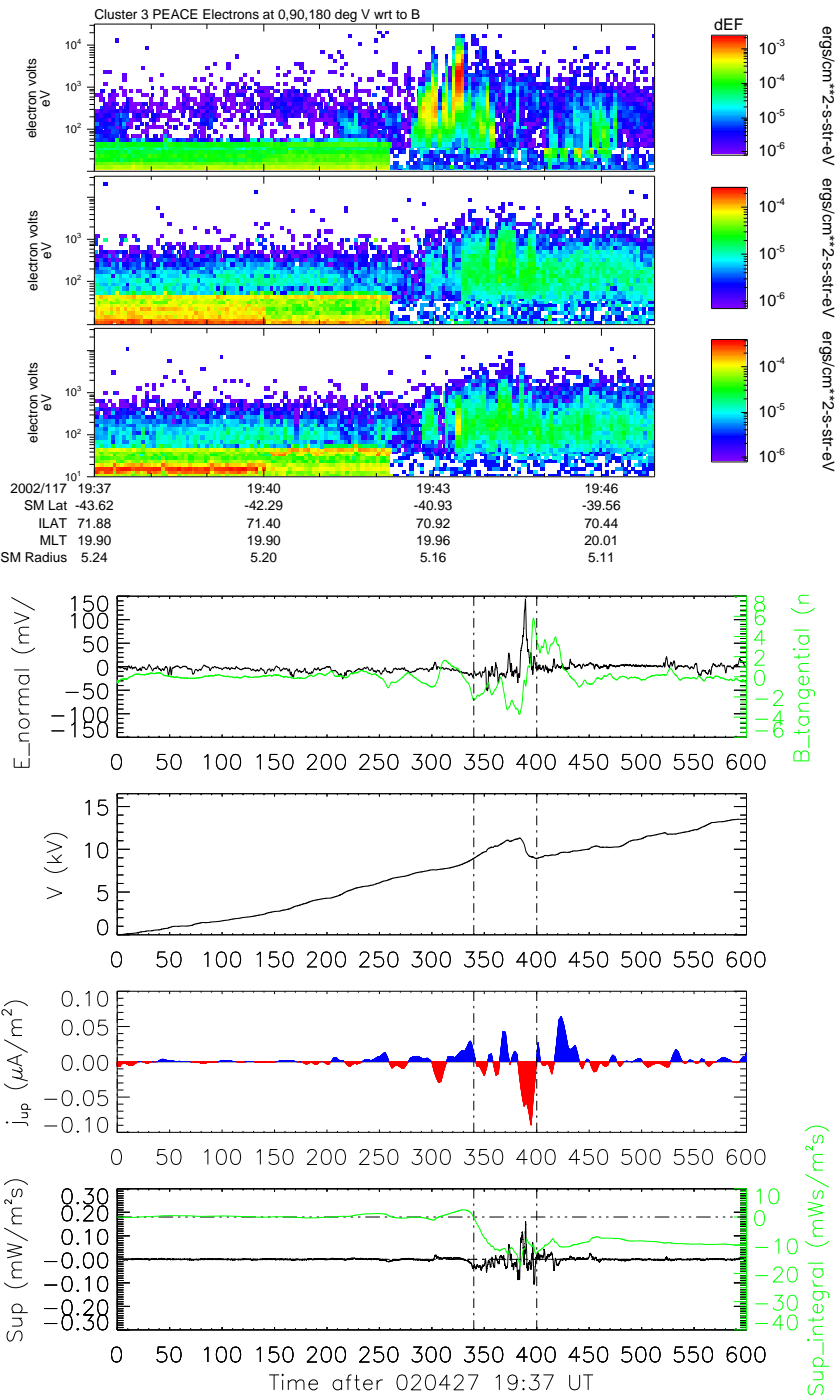

Fig. 10. 27 April 2002, s/c 3. The same panels as in Fig. 2.

region of electron flux enhancement around $375 \mathrm{~s}$, having a characteristic energy of $2-3 \mathrm{keV}$, was not observed in the passage by $\mathrm{s} / \mathrm{c} 1$, and is more intense and corresponds to a higher energy than the region of electron flux enhancement around $350 \mathrm{~s}$. The intensifications are consistent with intense down-going FAC peaks. Compared to the passage by s/c 1, a hill-structure has developed in the potential profile and there is also a larger potential difference over the whole interval, $13 \mathrm{kV}$ compared to $7.5 \mathrm{kV}$. Two smaller peaks are seen at the edges of the potential hill-structure. Note that none of the good correlated features in electric field structure, the potential peak and the intense region of electron flux at $375 \mathrm{~s}$ were observed in the passage by $\mathrm{s} / \mathrm{c} 1$, nor where the intense Poynting fluxes between 370 and 400 s observed in the passage by s/c 1 . The downward Poynting flux peak close to $380 \mathrm{~s}$, mapping to $27 \mathrm{~mW} / \mathrm{m}^{2}$, is well correlated with the main peak in the electric field and the main downward FAC peak of $10 \mu \mathrm{A} / \mathrm{m}^{2}$ (mapped) and most intense electron flux. Most of the Poynting flux is restricted to the region dominated by spatial variations in the electric and magnetic fields.
S/c 2: The large-scale electric pattern is fairly unchanged (Fig. 9), but a decrease in the amplitude of the peak can be seen and the peak has split into two peaks, and this is also true for the FAC pattern. The correlation between downward FAC peaks and the electric field peak is good. Significant variations compared to the passages by s/c 1 and 4 are observed in the Poynting flux. The main peak at $380 \mathrm{~s}$ is upward but the integrated Poynting flux is negative, hence the net Poynting flux is downward, having contributions from a downward Poynting flux peak at $300 \mathrm{~s}$ and another peak around $340 \mathrm{~s}$. The intense positive Poynting flux peak at $385 \mathrm{~s}$ is seen to be co-located with a downward FAC peak. The first of the minor peaks at the edges of the potential hill, observed in the passage by $\mathrm{s} / \mathrm{c} 4$, is not present in the data from this passage.

S/c 3: The electric field pattern is again roughly the same (the amplitude of the peak is higher) but the slope on the equatorward flank of the positive potential hill around $380 \mathrm{~s}$ is seen to be steeper than in the passage by s/c 2 (Fig. 10), corresponding to the increase in the electric field peak to $150 \mathrm{mV} / \mathrm{m}(1700 \mathrm{mV} / \mathrm{m}$ mapped). Also, the FAC pattern is fairly unchanged compared to the passages by the other s/c. The narrow region of intense electron flux at $390 \mathrm{~s}$ with characteristic energy of $2-3 \mathrm{keV}$ is more intense than what was observed by the other $\mathrm{s} / \mathrm{c}$ in this event and well correlated with the downward FAC peak, with the Poynting flux enhancement (with the clearest peak in the upward direction and with overall Poynting flux less downward dominated) and the intense electric field peak. The other region of enhanced electron flux has decreased significantly in intensity compared to the passage by s/c 1 and 4, and the first minor peak in the potential hill-structure is absent, as was also the case for the previous crossing. The correlations between the variations in the electric and magnetic fields, the potential and the electron flux, support the view that spatial variations dominate between 340 and $400 \mathrm{~s}$.

\subsection{Summary of observations}

Table 1 summarizes the events of 27 April 2002 and 19 May 2002, displaying Geocentric Distance (R), Corrected Geomagnetic Latitude (CGLat), Magnetic Local Time (MLT), $K_{p}$-index, substorm phase (determined from the AL-index), evolution of $\Delta V_{\perp}$, corresponding $\Delta V_{\|}$value (inferred from the characteristic energy of the up-going electrons observed by the PEACE instrument), Alfvén velocity $\left(V_{A}\right)$ and deviation of the current sheet orientation from east-west alignment, $\alpha . \Delta V_{\perp}$ represents the hill- (positive $\Delta V_{\perp}$ ) and valley- (negative $\Delta V_{\perp}$ ) shapes in the potential along the s/c trajectory. The $\Delta V_{\|}$and $\Delta V_{\perp}$ in Table 1 are given for comparison; if there is a positive U-shaped potential structure, these two should be similar. Since the $\Delta V_{\perp}$ is negative for the 19 May 2002 event, i.e. a potential well, this cannot be compared with a $\Delta V_{\|}$inferred from up-going electrons. The calculated Alfvén velocity is presented together with the ratio between the electric field component normal to the current sheet and the magnetic field tangential component, $E_{n} / B_{t}$, being average numbers for all the s/c. 


\section{Discussion and conclusions}

The intense electric field events presented here both took place in the local time evening sector, approximately at 20 MLT, in good agreement with statistical results from lower altitudes (Karlsson and Marklund, 1996), and from the relation between intense electric fields and low ionospheric background conductivity (Marklund et al., 1997). The magnitude of the maximum electric fields observed here are, if mapped to ionospheric altitudes, approximately 400 and $1500 \mathrm{mV} / \mathrm{m}$, for the two events, similar to reported maximum magnitudes observed at lower altitudes. Also, the two intense electric field events were encountered within the PSBL, which could be concluded from inspection of the PEACE electron data and CIS ion data (not shown here).

The question whether the structures encountered are spatial or temporal, or both, is fundamental for the understanding of their origin. Using the method described in the companion paper, it was found that spatial and temporal variations dominate in different regions. This is supported by the stability of the FAC and the electron distribution between the different $\mathrm{s} / \mathrm{c}$, indicating that the most intense variations in the two events described here were predominately spatial. However, in both events, a mix of both Alfvén waves and current sheets (FACs), sometimes well separated from each other and sometimes superposed, is observed. Alfvén waves could thus contribute to the variations also in the regions with dominating spatial structures. The observed spatial variations are quasi-static electric field structures associated with FAC current sheets and hence represent an energy transport between the magnetosphere and the ionosphere by FACs. Temporal variations correspond predominately to Alfvén waves and energy transport by Alfvénic Poynting flux. The most intense electric fields were found to be associated with spatial variations, whereas Alfvén wave-dominated regions were found to be characterized by less intense electric fields. An alternative interpretation of, what in this paper is considered to be, the quasi-static regions might be that Alfvén waves travel along the spatial boundaries and that the time delays are given by the encounters by the s/c with these waves. However, these Alfvén waves must have very low frequencies.

The most intense Poynting fluxes in these two events are directed downward and they are correlated with the intense quasi-static electric fields, with downward FAC peaks associated with up-going electrons and also with the acceleration potential structures. The Alfvén wave-dominated regions were typically associated with less intense electric fields and less intense Poynting fluxes. Thus, in the events studied here, there is a clear connection between the intense electric fields, FAC current sheets and strong downward Poynting fluxes.

Cluster offers the first opportunity to observe the stability of the different parameters on various time scales. Variations seen between the satellites indicate which parameters are more stable than others on the time scale given by the separation between the $\mathrm{s} / \mathrm{c}$ in the reference frame of the moving FAC sheet (typically 10-20s for the events studied here). The electric and magnetic field patterns, and the associated FAC pattern are qualitatively not so variable between the satellites, while the perpendicular potential is a parameter that displays some variations. The Poynting flux is seen to be concentrated to a few locations, whose positions remain the same, whereas the magnitudes show strong variations on the time scale of $10-50 \mathrm{~s}$. The variations in the electron energytime spectrograms between the s/c are generally fairly small, but they show enhancements for the up-going electrons consistent with the downward FAC.

In the event of 27 April 2002, the growth of a positive potential structure accelerating electrons away from Earth is seen. A good consistency was obtained between the characteristic energy of the up-going electrons, i.e. the acceleration potential $\triangle \mathrm{V}_{\|}$, inferred from PEACE electron spectrograms, and the calculated perpendicular potential $\triangle \mathrm{V}_{\perp}$ during this growth period, serving as evidence of quasi-static U- or Sshaped positive potential structures in the auroral return current region. The effective time lag between the crossings by $\mathrm{s} / \mathrm{c} 1$ and s/c 3 was $25 \mathrm{~s}$, taking into account the motion of the structure. During this period the acceleration potential grew by $2.4 \mathrm{kV}$, but a decay was not observed. In the study by Marklund et al. (2001), where the separations between the s/c were longer, the growth and decay of an acceleration potential took place within $200 \mathrm{~s}$. It was suggested by Marklund et al. (2001) that the lifetime is clearly related to the time it takes to evacuate the ionospheric electrons within the flux tube of the downward current, which depends on the FAC magnitude. Since the magnitudes of the FACs in the 27 April 2002 event are about half of that in Marklund et al. (2001), the expected lifetime of the acceleration potential would be more than $200 \mathrm{~s}$. However, the separation between the s/c is small and only growth is observed.

An interesting difference from the event discussed by Marklund et al. (2001) is that the intense electric field structures observed in the two events studied here are unipolar, not bipolar. This is somewhat unexpected since observations at lower altitudes mostly have shown divergent bipolar electric field structures. An S-shaped potential structure might explain the unipolar field structures.

There can be no doubt that both quasi-static structures and Alfvénic Poynting flux are important for the energy transport between the ionosphere and the magnetosphere. Schriver et al. (2003), for example, have shown that FAC and Alfvén waves both contribute to the energy transport into the auroral region. Where and under what circumstances each of the two is present and/or dominates is a question of importance and one that is subject to on-going work. The results from Polar discussed above pointed at the Alfvén wave Poynting flux as a major contributor to the powering of the aurora (Keiling et al., 2003), and Wygant et al. (2002) have shown that the Alfvén wave Poynting flux was the larger contributor in their events. The events investigated and presented here imply that the most intense electric field structures, associated with FACs and intense downward Poynting flux, were signatures of quasi-static acceleration structures. These events occurred in the return current region, while the referred work on 
Polar data is from the primary current region. This could be a reason for the discrepancy between the results. It has been shown (Keiling et al., 2001) that Alfvén wave aurora can be associated with aurora at the poleward edge of the PSBL. The results presented here indicate that intense electric fields near the polar cap can also be associated with quasi-static structures. The nature of the electric field structures may depend on whether they are encountered in the primary or return current region, which may explain the differences in observations.

The main conclusions drawn in this study of two intense electric field events are:

1. The energy transport between the magnetosphere and the ionosphere, in these two events (return current region), has contributions from both FACs and Alfvén wave Poynting fluxes.

2. The most intense electric fields were found to be quasistatic structures associated with moving quasi-static FAC current sheets. The Alfvén wave dominated regions were found to be associated with less intense electric fields. The most intense Poynting fluxes are downward directed and well correlated with the intense quasi-static electric fields, which implies that, for the events studied here at a geocentric distance of $5 R_{E}$ and during moderate geomagnetic activity, the quasi-static FACs represented the dominant contribution to the energy transport between the magnetosphere and the ionosphere in the return current region.

3. The FACs and the structure of the associated electron distributions tend to be fairly stable, while the perpendicular potential, correlated with the characteristic energy of the up-going electrons, shows more variations on the time scale between consecutive s/c crossings, of $10-40 \mathrm{~s}$. The Poynting flux peaks vary in magnitude, whereas their locations remain stable.

4. The observed growth of a positive potential structure accelerating electrons away from Earth during effectively $25 \mathrm{~s}$ gives a lower limit of the lifetime of an acceleration potential growing in magnitude.

5. Upward ions associated with negative potential peaks are observed which demonstrate that at least some negative U-shaped potential structures extend up to 5$5.5 R_{E}$. However, the most significant potential structures observed at this altitude are positive and associated with up-going accelerated electron beams as in the event reported by Marklund et al. (2001).
Acknowledgements. This work was supported by the Swedish National Space Board and the Royal Institute of Technology. One of the authors, S. Figueiredo, acknowledges the support of the Fundação para a Ciência e a Tecnologia (FCT) under the grant SFRH/BD/6211/2001.

Topical Editor T. Pulkkinen thanks H. Koskinen and A. Keiling for their help in evaluating this paper.

\section{References}

Escoubet, C., Russell, C., and Schmidt, R.: The Cluster and Phoenix missions, Space Sci. Rev., 79/1-2, 1-658, 1997.

Karlsson, T. and Marklund, G.: A statistically study of intense lowaltitude electric fields observed by Freja, Geophys. Res. Lett., 23, 1005-1008, 1996.

Karlsson, T., Marklund, G., Figueiredo, S., Johansson, T., and Buchert, S.: Separating spatial and temporal variations in auroral and magnetic fields by Cluster multipoint measurements, Ann. Geophys., 22, 7, 2463-2472, 2004.

Keiling, A., Wygant, J., Cattell, C., Johnson, M., Temerin, M., Mozer, F., Kletzing, C., Scudderm, J., Russell, C., Lotko, W., and Streltsov, A.: Large Alfvén wave power in the plasma sheet boundary layer during the expansion phase of substorms, Geophys. Res. Lett., 27, 3169-3172, 2000.

Keiling, A., Wygant, J., Cattell, C., Johnson, M., Temerin, M., Mozer, F., Kletzing, C., Scudder, J., and Russell, C.: Properties of large electric fields in the plasma sheet at 4-7 $R_{E}$ measured with Polar, J. Geophys. Res., 106, 5779-5798, 2001.

Keiling, A., Wygant, J., Cattell, C., Peria, W., Parks, G., Temerin, M., Mozer, F., Russell, C., and Kletzing, C.: Correlation of Alfvén wave Poynting flux in the plasma sheet at $4-7 R_{E}$ with ionospheric electron energy, J. Geophys. Res., 107, A7 SMP 24 $1-13,2002$.

Keiling, A., Wygant, J., Cattell, C., Mozer, F., and Russell, C.: The global morphology of wave Poynting flux: Powering the aurora, Science, 299, 383-386, 2003.

Lotko, W., Streltsov, A., and Carlson, C.: Discrete auroral arc, electroststic shock and suprathermal electrons powered by dispersive, anomalously resistive field line resonance, Geophys. Res. Lett., 25, 4449-4452, 1998.

Marklund, G., Karlsson, T., and Clemmons, J.: On low-altitude particle acceleration and intense electric fields and their relation to black aurora, J. Geophys. Res., 102, 17 509-17 522, 1997.

Marklund, G., Ivchenko, N., Karlsson, T., Fazakerley, A., Dunlop, M., Lindquist, P.-A., Buchert, S., Owen, C., Taylor, M., Vaivalds, A., Carter, P., André, M., and Balogh, A.: Temporal evolution of the electric field accelerating electrons away from the auroral ionosphere, Nature, 414, 724-727, 2001.

Ostgaard, N., Vondrak, R., Gjerlov, J., and Germany, G.: A relation between the energy deposition by electron precipitaion and geomagnetic indicies during substorms, J. Geophys. Res., 107, A9 SMP 16 1-7, 2002.

Samson, J., Cogger, L., and Pao, Q.: Observations of field line resonances, auroral arcs, and auroral vortex structures, J. Geophys. Res., 101, 17 373-17 383, 1996.

Samson, J., Rankin, R., and Tikhonchuk, V.: Optical signatures of auroral arcs produced by field line resonances: comparison with satellite observations and modeling, Ann. Geophys., 21, 933945, 2003. 
Schriver, D., Ashour-Abdalla, M., Strangeway, R., Richard, R., Klezting, C., Dotan, Y., and Wygant, J.: FAST/Polar conjunction study of field-aligned auroral acceleration and corresponding magnetotail drivers, J. Geophys. Res., 108, A9 COA 21 1-21, 2003.

Wygant, J., Keiling, A., Cattell, C., Johnson, M., Lysak, R., Temerin, M., Mozer, F., Kletzing, C., Scudder, J., Peterson, W., Russell, C., Parks, G., Brittnacher, M., Germany, G., and Spann, J.: Polar spacecraft based comparisons of intense electric fields and Poynting flux near and within the plasma sheet-tail boundary to UVI images: An energy source for the aurora, J. Geophys. Res., 105, 18 675-18 692, 2000.
Wygant, J., Keiling, A., Cattell, C., Lysak, R., Temerin, M., Mozer, F., Kletzing, C., Scudder, J., Streltsov, V., Lotko, W., and Russell, C.: Evidence for kinetic Alfvén waves and parallel electron energization at $4-6 R_{E}$ altitudes in the plasma sheet boundary layer, J. Geophys. Res., 107, A8 SMP 24 1-13, 2002. 